\title{
1 When to declare successful eradication of an invasive predator
}

2

3 Tracy M. Rout ${ }^{1,2 *}$, Roger Kirkwood ${ }^{3}$, Duncan R. Sutherland ${ }^{3}$, Stuart Murphy ${ }^{4}$, and Michael A.

$4 \quad$ McCarthy $^{1}$

$5{ }^{1}$ School of Botany, The University of Melbourne, Parkville 3010 Australia.

$6 \quad{ }^{2}$ School of Biological Sciences, The University of Queensland, St Lucia 4067 Australia.

$7 \quad{ }^{3}$ Research Department, Phillip Island Nature Parks, PO Box 97 Cowes, Victoria 3922 Australia.

$8{ }^{4}$ Environment Department, Phillip Island Nature Parks, PO Box 97 Cowes, Victoria 3922

9 Australia.

10

11 *corresponding author: Tracy M. Rout, tmrout@unimelb.edu.au, School of Biological Sciences,

12 The University of Queensland, St Lucia 4067 Australia.

14 Short title: When to declare successful eradication

15 Word count: 3559, Abstract: 272. 


\section{Abstract}

2 Imperfect detection methods make it difficult to tell whether an invasive species has been

3 successfully eradicated. However, management cannot continue indefinitely when individuals

4 are no longer detected - at some point efforts must be reduced or ceased entirely. The risks of

5 mistakenly inferring that an eradication attempt has been successful can be high: the species

6 can bounce back and even expand its range, causing environmental and economic damage and

7 rendering the initial eradication campaign redundant. This decision problem, balancing the risks

8 of declaring eradication prematurely with the costs of continued management, is currently

9 being contemplated by managers of the fox eradication program on Phillip Island, in Victoria,

10 Australia. We used a Bayesian catch-effort model to analyse data on the number of foxes

11 removed and sighted using different methods. We estimate that there were 11 foxes remaining

12 on Phillip Island as of end of June 2012. Baiting was the most effective method for removing

13 foxes per person-hour invested, and spotlighting was the most effective method for sighting

14 foxes without removal. We then projected forward into the future, assuming management

15 effort continues at current levels but no further foxes are detected (removed or sighted). Under

16 this scenario, the mean estimate for the number of foxes remaining drops below a single fox

17 after three years with no detections, and the probability that eradication has been successful is

18 0.69. This is the optimal time to declare eradication, given our estimated cost of declaring

19 eradication prematurely. This framework indicates the minimum number of years for which

20 management of foxes on the island must continue, and allows decision-makers to assess the

21 trade-offs involved in any decision to declare eradication. 
1 Keywords: Bayesian statistics, catch-effort models, decision theory, detection, monitoring,

2 Vulpes vulpes.

3

\section{Introduction}

5 The majority of documented extinctions in the last 500 years have occurred on islands, and of

6 these, most vertebrates were driven to extinction by predation (Sax \& Gaines 2008). When non-

7 native predators invade predator-naive island systems, the effect on native wildlife can be

8 devastating (Blackburn et al. 2004; Jones et al. 2008). This has motivated numerous campaigns

9 to eradicate feral predators from islands (Towns \& Broome 2003; Nogales et al. 2004; Howald

10 et al. 2007; Oppel et al. 2011).

11 Eradication of invasive species presents a dual challenge for managers. Firstly, it is difficult to

12 reduce a population to the point of eradication. Secondly, due to imperfect detection, it is

13 difficult to know if successful eradication has been achieved (Regan et al. 2006). When

14 monitoring and management stop detecting individuals, there comes a point when efforts must

15 be reduced or ceased entirely. However, the risks of mistakenly assuming success can be high:

16 the species can bounce back and render the eradication attempt redundant (Solow et al. 2008),

17 or even escape the delimited area (Regan et al. 2006). Given these risks, and the costs of

18 continued monitoring and management, how certain should managers be before declaring an

19 eradication campaign successful? 
1 This question is currently being contemplated by managers of the invasive fox eradication

2 program on Phillip Island, Australia. Phillip Island is an approximately $100 \mathrm{~km}^{2}$ island off the

3 coast of Victoria and is connected by a bridge to the mainland. Red foxes (Vulpes vulpes) were

4 first seen on the island in the early 1900s, and threaten much of the island's wildlife (Lade et al.

5 1996). In particular, the little penguin (Eudyptula minor) population has been reduced from ten

6 colonies to only one colony, mainly due to fox predation (Dann 1992). This remaining colony is a

7 popular tourist attraction, with almost 500,000 visitors per year paying to watch a nightly

8 Penguin Parade (when penguins return to their nests after sunset, Phillip Island Nature Parks

$92011)$.

10 Five different methods have been used to find and remove foxes on Phillip Island: leg-hold

11 trapping, baiting, hunting, spotlighting, and den searches. Since 1986, fox control has been

12 conducted over most of the island, including all public reserves and some agricultural

13 properties. Over this time, the number of hours invested in each management method and the

14 number of foxes removed and sighted with each method have been recorded (Fig. 1). In 2006,

15 the management strategy shifted from controlling fox damage to island-wide eradication, and

16 management coverage was expanded to include most private land. Since then, the number of

17 foxes removed and sighted has dropped, while at the same time the amount of management

18 effort stabilised (Fig. 1). Managers expect that in the near future they will stop detecting foxes,

19 and are eager to plan ahead by determining how long management should continue when

20 foxes are no longer detected. How long should they keep managing and searching before

21 declaring the eradication program a success? 
1 In this study, we use a Bayesian catch-effort model (e.g., Ramsey, Parkes \& Morrison 2009;

2 Ramsey et al. 2011) to estimate the number of foxes that were present on Phillip Island in the

3 years from 1986 to 2012, and the effectiveness of each of the five management methods in

4 removing and sighting foxes. We then project ahead to consider the possibility that no foxes are

5 detected in the future, and estimate the future population size and the probability of extinction

6 (i.e., the probability of successful eradication). By considering the costs of management and the

7 risks of stopping management prematurely, we determine the best time to declare foxes

8 eradicated and redirect management effort (Regan et al. 2006; Ramsey, Parkes \& Morrison

9 2009; Rout, Salomon \& McCarthy 2009). Our analysis provides a framework for better decision-

10 making about the termination of this eradication campaign, and provides a case study for

11 managers of other invasive species eradication programs.

13 Methods

14 Estimating population size

15 Effort and fox removal and sighting data were compiled in financial years, starting on the 1st of 16 July and ending on the $30^{\text {th }}$ of June. Baiting and leg-hold trapping require one staff member to 17 place the poison baits, or the treadle and soft jaw snare traps. Spotlighting requires two staff 18 members and involves the detection and opportunistic shooting of foxes at night. Hunting 19 requires a team of six to eight shooters with hounds, while den searching requires the same

20 number to find and fumigate fox dens. Because of these different staff requirements, we 21 measured effort by the number of person-hours spent on each management method in each 
1 year. We modelled the probability of removing a fox with each method $j$ in year $t$ as a function

2 of the amount of effort invested in that method:

$3 \quad p_{t, j}=1-e^{-a_{j} g_{t, j}}$,

4 where $a_{j}$ describes the estimated effectiveness of method $j$, and $g_{t, j}$ is the amount of effort (in

5 person-hours) invested in method $j$ at time $t$. For hunting and spotlighting, some foxes were

6 only sighted and not removed. For these methods, we also modelled the probability of sighting

7 a fox as a function of the amount of effort invested:

$8 \quad q_{t, j}=1-e^{-b_{j} g_{t, j}}$,

9 where $b_{j}$ is the method's estimated effectiveness in sighting foxes.

10 The numbers of foxes removed by leg-hold trapping, spotlighting and hunting were recorded

11 directly from collected carcasses. The number of removals through baiting was estimated

12 indirectly: before the eradication program began in 2006 it was inferred by the number of baits

13 taken and the number of foxes identifiable by their prints before and after the baiting, while

14 after 2006 it was assumed that 2.5 bait takes in an area meant one fox had been removed

15 (Kirkwood et al. in review). This guideline is based on published estimates ranging from 1.6 to

163.0 bait takes per fox (Trewhella et al. 1991; Thompson \& Fleming 1994), to account for

17 multiple takes by one fox, regurgitation, and caching without consumption (Van Polanen Petel

18 et al. 2001; Saunders \& McLeod 2007). The number of removals through den searches was also

19 estimated indirectly before 2006 by assuming each fumigated den contained a vixen and four

20 cubs. This is the maximum number of foxes that team members could recall finding in an 
1 excavated den, and so is likely to overestimate the average number of foxes in a den (Kirkwood

2 et al. in review). After 2006 the dens were excavated to record the actual number of foxes

3 removed (Kirkwood et al. in review).

4 For each management method $j$, the number of removals in each year $t$ was modelled as a

5 binomial process:

$6 \quad n_{t, j} \sim \operatorname{Bin}\left(p_{t, j}, \lambda N_{t-1}\right)$,

7 where $p_{t, j}$ is the probability of removing a fox at time $t$ with method $j, \lambda$ is the annual growth

8 rate of the population, and $N_{t-1}$ is the number of foxes in the population at the start of time $t$.

9 Similarly, the number of sightings (without removal) using method $j$ in year $t$ is given by:

$10 \quad m_{t, j} \sim \operatorname{Bin}\left(q_{t, j}, \lambda N_{t-1}\right)$.

11 The population growth rate $\lambda$ incorporates reproduction and natural mortality. Due to the

12 relative isolation of Phillip Island, immigration is minimal ( 3 immigrants found in 14 years, Berry

13 \& Kirkwood 2010), and we assumed the same for emigration. We assumed population changes

14 occur before removals and sightings in each year (Fig. 2), based on the fact that foxes have a

15 distinct breeding season with reproduction peaking in September and October on Phillip Island

16 (Berry \& Kirkwood 2010).

17 The number of foxes on the island at the end of year $t$ is then estimated as:

18

$$
N_{t}=\lambda N_{t-1}-\rho_{t},
$$

19 where $\rho_{t}=\sum_{j} n_{t, j}$, the total number of foxes removed in year $t$. 
1 The model inputs were yearly data on the number of fox removals using each method $\left(n_{t, j}\right)$, the

2 number of fox sightings using each method $\left(m_{t, j}\right)$, and the amount of effort invested in each

3 method $\left(g_{t, j}\right)$. We used the model to estimate posterior distributions for the fox population size

4 in each year $\left(N_{t}\right)$ and the effectiveness of each method in removing and sighting foxes $\left(a_{j}, b_{j}\right)$.

5 We ran the analysis using a population growth rate of $\lambda=1.32$, the estimated growth rate of a

6 low density fox population in Western Australia (Marlow et al. 2000; Devenish Nelson et al.

7 2010). This population was not harvested before being sampled, making this growth rate

8 indicative of a natural population growth rate independent of removals. We used a uniform

9 prior distribution $N_{0} \sim U(1,1600)$ for the number of foxes on the island before data began to be

10 systematically collected in 1986 . The upper limit of 1600 foxes was derived by considering the

11 size of Phillip Island $\left(100 \mathrm{~km}^{2}\right)$ and the maximum fox density estimated in Australia (16

12 foxes $/ \mathrm{km}^{2}$ ) (Saunders et al. 1995; Marks \& Bloomfield 1999). We used uniform prior

13 distributions for the effectiveness parameters, $b_{j} \sim U(0,10)$, and generated posterior

14 distributions for the population size $N_{t}$ and the effectiveness parameters $b_{j}$ using Markov chain

15 Monte Carlo (MCMC) sampling in OpenBUGS (Lunn et al. 2009). To achieve convergence, we

16 ran 6 chains for 100,000 iterations with a thinning rate of 10 , and then generated posterior

17 distributions from a further 100,000 iterations.

18 To assess the fit of this model, we compared the number of removals and sightings predicted by

19 the mean estimates of $N_{t}, a_{j}$ and $b_{j}$ for each method in each year, with the actual number of

20 foxes removed and sighted that year. To see how well our model outputs predicted the actual

21 removals and sightings, we analysed these data with a standardised major axis (SMA)

22 regression. Unlike the ordinary least squares method, which accounts only for error in the 
1 response variable, SMA regression accounts for error in both the response and predictor

2 variables.

$4 \quad$ Planning for eradication

5 Next we explored scenarios where management continues into the future, but no further foxes

6 are detected (i.e., foxes are neither removed nor sighted). We ran the catch-effort model with

7 the same parameter values and prior distributions as previously, but we created effort, removal

8 and sighting data that continued up to ten years into the future. For the amount of effort

9 allocated to each method in each year, we used the yearly average number of person-hours

10 spent on each method over the eradication program (2006-2012): $g_{t, \text { leg-hold trapping }}=404.03$,

$11 g_{t, \text { baiting }}=349.77, g_{t, \text { hunting }}=187.45, g_{t, \text { spotlighting }}=436.58, g_{t, \text { den searches }}=311.50$. For each year from

$12 T=2013$ to 2022 , we ran a separate model using real data for $1987-2012$, and generated data

13 (with no detections) from 2013 onwards. We calculated the posterior distribution for the

14 population size in the final year $N_{T}$, and the probability of successful eradication $P\left(N_{T} \leq 1\right)$. We

15 ran the analyses using the same prior distributions, and for the same number of iterations as

16 previously.

17 The best time to declare successful eradication and stop management depends not only on the

18 probability that eradication has been successful, but also on the cost of management and the

19 consequences of declaring eradication prematurely. To determine the best time to declare

20 successful eradication under this scenario, we calculated the net expected cost of declaring

21 foxes eradicated after $d$ years with no removals or sightings (Regan et al. 2006): 
1

2 where $C_{m}$ is the yearly cost of management, $1-P\left(N_{T} \leq 1 \mid d\right)$ is the probability that foxes are still

3 present despite $d$ years without detections, and $C_{p}$ is the cost of declaring foxes eradicated

4 when they are still present. The optimal year to declare eradication $\left(d^{*}\right)$ is the year in which the

5 expected cost of doing so is the lowest (Regan et al. 2006).

6 Managing foxes on Phillip Island currently costs around AU\$160,000 per year. If foxes were

7 declared eradicated, and management was stopped while foxes were still present, the

8 population could rebound to levels seen before the eradication program began in 2006. Further

9 eradication attempts would then have to re-do the work the eradication program has

10 accomplished over the past six years, at a total cost of six times the current yearly management

11 cost. We used this as a baseline estimate for $C_{p}(A \cup \$ 960,000)$. However, mindful of uncertainty

12 in estimating the consequences of declaring eradication prematurely, we found the optimal

13 solution for a range of possible costs.

\section{Results}

16 According to our model, at the end of June 2012 there were an estimated 11 foxes remaining

17 on Phillip Island (mean $=11.4,95 \%$ credible interval $=[4.6-19.6])$. Our analysis shows that in

181987 there were around 125 foxes on the island, and this rose to over 200 foxes in 1996 (Fig. 3).

19 Numbers have decreased since 2004, and have continued to decrease with the eradication

20 program implemented in 2006 (Fig. 3). The credible intervals around these population 
1 estimates are very narrow (Fig. 3), due to our use of a point estimate for the annual population

2 growth rate $\lambda$.

3 Our estimates of management effectiveness show that baiting is the most effective method for

4 removing foxes per person-hour of effort $\left(a=6.9 \times 10^{-4}\right)$, followed by leg-hold trapping and

5 spotlighting (both with $a=2.4 \times 10^{-4}$ ) (Fig. 4a). The $95 \%$ credible intervals on the effectiveness

6 of management for fox removal only overlapped for the equally-effective leg-hold trapping and

7 spotlighting. Spotlighting was the most effective method for sighting foxes without removal $(b=$

$86.8 \times 10^{-4}$ ), although a few sightings without removal occurred while hunting (Fig. 4b). Again,

9 the $95 \%$ credible intervals did not overlap for these methods. The relationship between the

10 model-predicted and actual number of fox removals and sightings was very close to a one-to-

11 one relationship (Fig. 5), with an estimate of $m=1.01$ for the slope of the line (95\% confidence

12 interval $=[0.90-1.1])$ and $c=-0.04$ for the $y$-intercept (95\% confidence interval $=[-1.9-1.8]$ ).

13 If management continues as under the eradication program for ten years from July 2012, but

14 no further foxes are detected beyond 2012, the probability that foxes will have been

15 successfully eradicated in 2022 is 0.96 (Fig. 6). The mean estimate of population size drops

16 below a single fox after three years of management without detection, with lower and upper

$1795 \%$ credible bounds of 0.03 and 2.95 respectively. The probability of successful eradication at

18 this point (i.e., the probability that $N_{t} \leq 1$ ) is 0.69 (Fig. 6).

19 The net expected cost of declaring foxes eradicated is quite high in 2013-14 (Fig. 7), due to the

20 cost of declaring eradication prematurely. As the number of years without detection increases,

21 this cost decreases and then increases again as the yearly costs of management accumulate 
1 (Fig. 7). Given our best estimates of the yearly cost of management $\left(C_{m}=\mathrm{AU} \$ 160,000\right)$ and the

2 cost of declaring eradication prematurely $\left(C_{p}=6 C_{m}\right)$, the optimal time to declare foxes

3 eradicated is in 2015, after three years of management without detections. The net expected

4 cost of this optimal decision is AU\$619,616.

5 If the cost of declaring eradication prematurely is higher than our baseline assumption, it is

6 optimal to continue management for longer (Fig. 8). It can be optimal to continue management

7 for up to nine years with no detections if the cost of declaring eradication prematurely is 100

8 times the yearly cost of management (for $C_{m}=\mathrm{AU} \$ 160,000, C_{p}=\mathrm{AU} \$ 16$ million). The net

9 expected cost of the optimal decision increases correspondingly as the cost of declaring

10 eradication prematurely increases (Fig. 8).

\section{Discussion}

13 It can take years of management and searching to be reasonably confident in the success of an

14 eradication program. For foxes on Phillip Island, the probability of success is greater than 0.9

15 after seven years without detection, and greater than 0.95 after nine years. This estimate of

16 success may be optimistic, because management will select for wary foxes that avoid baits, leg-

17 hold traps, and other management activities, effectively reducing detection probabilities and

18 management efficacy over time (Kirkwood et al. in review). However, this decrease could be

19 offset by improvements in management efficiency through time as staff gain experience and

20 skills. 
1 Our past and present population estimates are within a realistic range and are consistent with a

2 previous analysis of the fox population on Phillip Island. Berry \& Kirkwood (2010) compiled

3 biological information of foxes killed on the island between 1994 and 2008, such as their age,

4 size, gender, and reproductive status, and used this to estimate the minimum population size

5 over that time. Their analysis found that the population size was $>140$ foxes during the late

6 1990s, with a minimum estimate of approximately 90 foxes during the early 2000s (Berry \&

7 Kirkwood 2010). Our model replicates this trend, with an estimated peak of 204 foxes in 1996,

8 decreasing to 162 in 1999, and stabilising around 140 foxes in the early 2000s (Fig. 3).

9 In developing a reasonable model of this fox population, we have enabled further questions

10 about its management to be answered. In this study, we considered a future where

11 management continues in the same way it has been under the eradication program. However,

12 further work could also shed some light on how the different management methods should be

13 prioritised in the future. For example, we could examine the best time to switch from the

14 current management strategy to a monitoring only strategy, or the best way to allocate a

15 management budget between the different methods (McCarthy et al. 2010).

16 We used an exponential function to describe the relationship between the probability of

17 removing or sighting a fox with a given method and the number of hours spent applying that

18 method. This exponential function is based on assumed random and independent encounters

19 where all individuals have the same rate of detection (Moore et al. 2011). If the rate varies

20 among individuals, the probability of missing an individual will decrease more slowly as the

21 amount of effort invested is increased, reducing the marginal benefit of increased management 
1 effort (McCarthy et al. 2010). Our estimates of the efficacy of baiting and den searching are

2 influenced by assumptions made about bait take and den fumigation when data were recorded

3 (see Methods). For an eradication decision to be robust to uncertainty in the form or

4 parameterisation of the detection function, it is best to continue management for longer than

5 predicted by the optimal solution (Rout, Thompson \& McCarthy 2009).

6 Unfortunately, data on the Phillip Island fox population were not sufficient to estimate the

7 annual population growth rate in addition to population size and management efficacy, even

8 with an informative prior distribution. We instead used a point estimate from a similar fox

9 population, which resulted in a very narrow credible interval around estimated fox numbers.

10 While the mean estimates and trends shown by our model seem reasonable for the population

11 on Phillip Island, they are influenced by this estimate of growth rate. A higher growth rate

12 would lead to lower population estimates and higher estimates of management efficacy; vice

13 versa for a lower growth rate. As more data are collected on this population into the future, it

14 may become possible to estimate the population growth rate in addition to other model

15 parameters.

16 Our estimate of the consequences of declaring eradication prematurely focuses on the

17 possibility that the fox population will rebound to previous levels, and the management cost of

18 reducing the population back down to an eradicable size. This is a baseline estimate only, as

19 there are other possible consequences of declaring eradication prematurely that we have not

20 considered, for example, decreased tourism revenue and environmental impacts. Reductions in

21 the fox population since 2006 have been correlated with increases in several species of ground- 
1 dwelling birds, for example, the abundance of masked lapwings (Vanellus miles) seen across the

2 island has increased, as have the number of hooded plover (Thinornis rubricollis) eggs hatched

3 and fledged (Phillip Island Nature Parks 2011, Kirkwood et al. in review). Also, few little penguin

4 deaths due to fox predation have been recorded since 2008. In 2010-11 the average number of

5 little penguins crossing the beach nightly during the Penguin Parade was the highest since

6 counting began in 1977 (Phillip Island Nature Parks 2011, Sutherland \& Dann submitted) and

7 the population on Phillip Island has doubled since the 1980s (Harris \& Bode 1981; Sutherland \&

8 Dann 2012). These increases in birds that are vulnerable to fox predation likely resulted from

9 multiple factors, but their correlation with each other and with reductions in the fox population

10 are highly suggestive. If we consider these as environmental benefits of fox control, they could

11 be reversed if fox eradication is declared prematurely and the population rebounds.

12 These types of consequences are extremely difficult to estimate in a way that is

13 commensurable with the monetary cost of management. However, given these risks, managers

14 may think it appropriate to spend longer than three years managing without detection before

15 declaring successful eradication. We do not intend this framework to dictate precisely when to

16 declare eradication, but rather to provide a tool for managers to uncover the hidden trade-offs

17 implicit in any decision to declare eradication and to assess whether these trade-offs are

18 acceptable to the decision-maker.

19 This paper joins two recent island case studies (Ramsey, Parkes \& Morrison 2009; Ramsey et al.

20 2011) in combining quantitative modelling with a simple decision framework to provide

21 decision support for real-world eradication problems. The conservation value and isolation of 
1 island ecosystems make them a high priority for eradication campaigns, and thus there are

2 many other island case studies to which this simple population model could be applied. The

3 eradication of "open" mainland populations could also be analysed by incorporating estimated

4 rates of immigration and emigration into the population model. Increased application of these

5 methods will facilitate more informed and transparent decisions about eradication campaigns,

6 which will ultimately maximise the chance of conserving vulnerable biodiversity.

8 Acknowledgements

9 We acknowledge the long-term efforts of the fox control team on Phillip Island. TMR was

10 supported by an Australian Research Council (ARC) APD Fellowship (DP110101499), and MAM

11 was supported by an ARC Future Fellowship and the ARC Centre of Excellence for

12 Environmental Decisions.

14 Literature cited

15 Berry, O. \& Kirkwood, R. (2010). Measuring recruitment in an invasive species to determine eradication potential. J. Wildl. Mgmt. 74, 1661-1670.

17 Blackburn, T.M., Cassey, P., Duncan, R.P., Evans, K.L. \& Gaston, K.J. (2004). Avian extinction and mammalian introductions on oceanic islands. Science. 305, 1955-1958.

19 Dann, P. (1992). Distribution, population trends and factors influencing the population size of 20 little penguins Eudyptula minor on Phillip Island, Victoria. Emu. 91, 263-272. 
1 Devenish Nelson, E.S., Harris, S., Soulsbury, C.D., Richards, S.A. \& Stephens, P.A. (2010). Uncertainty in population growth rates: determining confidence intervals from point estimates of parameters. PLOS ONE. 5, e13628.

4 Harris, M.P. \& Bode, K.G. (1981). Populations of little penguins, short-tailed shearwaters and other seabirds on Phillip Island, Victoria, 1978. Emu. 81, 20-28.

6 Howald, G., Donlan, C.J., Galvan, J.P., Russell, J.C., Parkes, J., Samaniego, A., Wang, Y., Veitch,

7 D., Genovesi, P., Pascal, M., Saunders, A. \& Tershy, B. (2007). Invasive rodent eradication on islands. Conserv. Biol. 21, 1258-1268.

9 Jones, H.P., Tershy, B.R., Zavaleta, E.S., Croll, D.A., Keitt, B.S., Finkelstein, M.E. \& Howald, G.R. (2008). Severity of the effects of invasive rats on seabirds: a global review. Conserv. Biol.

12 Kirkwood, R., Sutherland, D., Murphy, S. \& Dann, P. (in review). Lessons from long-term predator control - a case study with the red fox, Wildlife Res.

Lade, J.A., Murrray, N.D., Marks, C.A. \& Robinson, N.A. (1996). Microsatellite differentiation between Phillip Island and mainland Australian populations of the red fox Vulpes vulpes. Mol. Ecol. 5, 81-87.

Lunn, D., Spiegelhalter, D., Thomas, A. \& Best, N. (2009). The BUGS project: evolution, critique and future directions. Stat. Med. 28, 3049-3067.

Marks, C.A. \& Bloomfield, T.E. (1999). Distribution and density estimates for urban foxes (Vulpes vulpes) in Melbourne: implications for rabies control. Wildl. Res. 26, 763-775. 
1 Marlow, N.J., Thomson, P.C., Algar, D.A., Rose, K., Kok, N.E. \& Sinagra, J.A. (2000). Demographic

2

3

4 characteristics and social organisation of a population of red foxes in a rangeland area in Western Australia. WildI. Res. 27, 457-464.

McCarthy, M.A., Thompson, C.J., Hauser, C., Burgman, M.A., Possingham, H.P., Moir, M.L., Tiensin, T. \& Gilbert, M. (2010). Resource allocation for efficient environmental management. Ecol. Lett. 13, 1280-1289.

Moore, J.L., Hauser, C.E., Bear, J.L., Williams, N.S.G. \& McCarthy, M.A. (2011). Estimating detection-effort curves for plants using search experiments. Ecol. Appl. 21, 601-607.

Nogales, M., Martin, A., Tershy, B.R., Donlan, C.J., Veitch, D., Puerta, N., Wood, B. \& Alonso, J. (2004). A review of feral cat eradication on islands. Conserv. Biol. 18, 310-319.

Oppel, S., Beaven, B.M., Bolton, M., Vickery, J. \& Bodey, T.W. (2011). Eradication of invasive mammals on islands inhabited by humans and domestic animals. Conserv. Biol. 25, 232240.

Phillip Island Nature Parks (2011). Annual Report 2010-11. Available from http://www.penguins.org.au/information-downloads.

Ramsey, D.S.L., Parkes, J. \& Morrison, S.A. (2009). Quantifying eradication success: the removal of feral pigs from Santa Cruz Island, California. Conserv. Biol. 23, 449-459.

Ramsey, D.S.L., Parkes, J.P., Will, D., Hanson, C.C. \& Campbell, K.J. (2011). Quantifying the success of feral cat eradication, San Nicolas Island, California. New Zeal. J. Ecol. 35, 163174.

Regan, T.J., McCarthy, M.A., Baxter, P.W.J., Panetta, F.D. \& Possingham, H.P. (2006). Optimal eradication: when to stop looking for an invasive plant. Ecol. Lett. 9, 759-766. 
1 Rout, T.M., Salomon, Y. \& McCarthy, M.A. (2009). Using sighting records to declare eradication of an invasive species. J. Appl. Ecol. 46, 110-117.

3 Rout, T.M., Thompson, C.J. \& McCarthy, M.A. (2009). Robust decisions for declaring eradication 4 of invasive species. J. Appl. Ecol. 46, 782-786.

5 Saunders, G., Coman, B.J., Kinnear, J. \& Braysher, M. (1995). Managing Vertebrate Pests: Foxes. $6 \quad$ Australian Government Publishing Service, Canberra.

7 Saunders, G. \& McLeod, L. (2007). Improving fox management strategies in Australia. Bureau of

9 Sax, D.F. \& Gaines, S.D. (2008). Species invasions and extinction: the future of native

11 Solow, A.R., Seymour, A., Beet, A. \& Harris, S. (2008). The untamed shrew: On the termination 12 of an eradication programme for an introduced species. J. Appl. Ecol. 45, 424-427.

13 Sutherland, D.R. \& Dann, P. (2012). Improving accuracy of population size estimates for burrow nesting seabirds. Ibis. 154, 488-498.

15 Sutherland, D.R. \& Dann, P. (submitted). Population trends in a substantial colony of little

17 Thompson, P. C. , Fleming, P. J. S. (1994). Evaluation of the efficacy of 1080 poisoning of red foxes using visitation to non-toxic baits as an index of fox abundance. Wildl. Res., 21: 27- 
1 Trewhella, W., Harris, S., Smith, G. C. \& Nadan, A. K. (1991). A field trial evaluating bait uptake $2 \quad$ by an urban fox (vulpes vulpes) population. J. Appl. Ecol., 28: 454-466.

3 Van Polanen Petel, A., Marks, C. \& Morgan, D. (2001). Bait palatability influences the caching 4 behaviour of the red fox (vulpes vulpes). Wildl. Res., 28: 395-401. 
1 Figures

2 a)

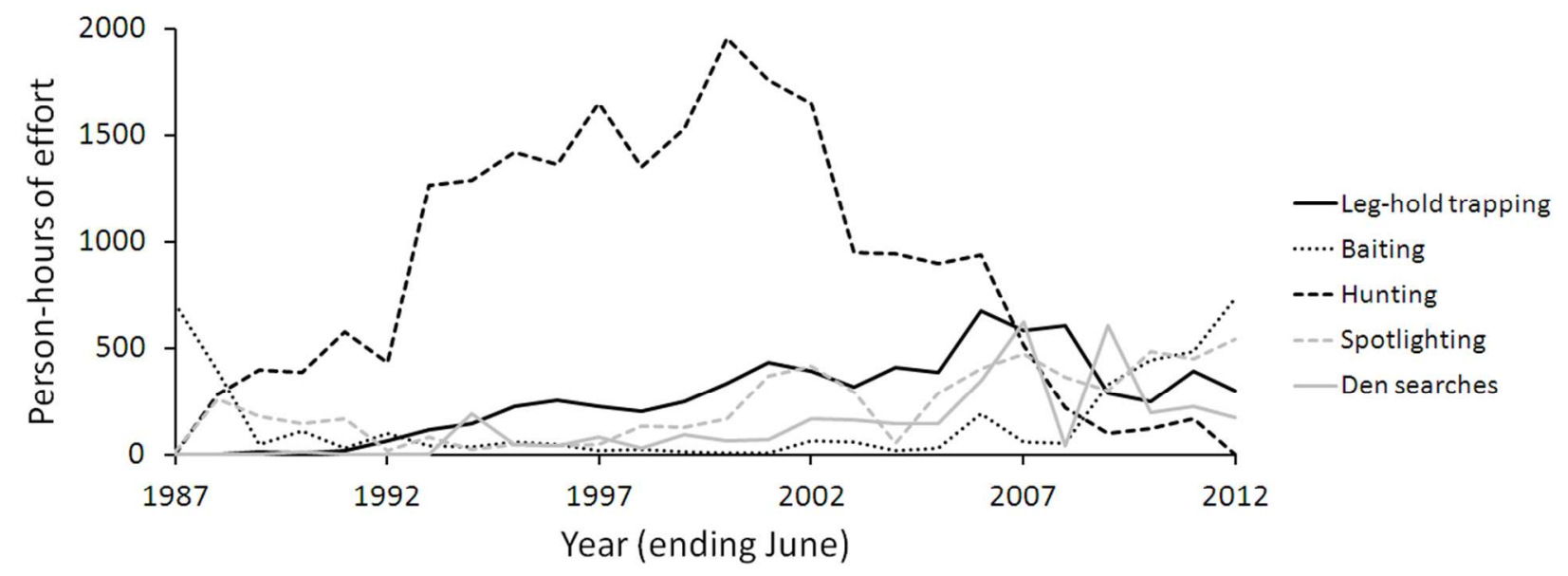

3

4 b)

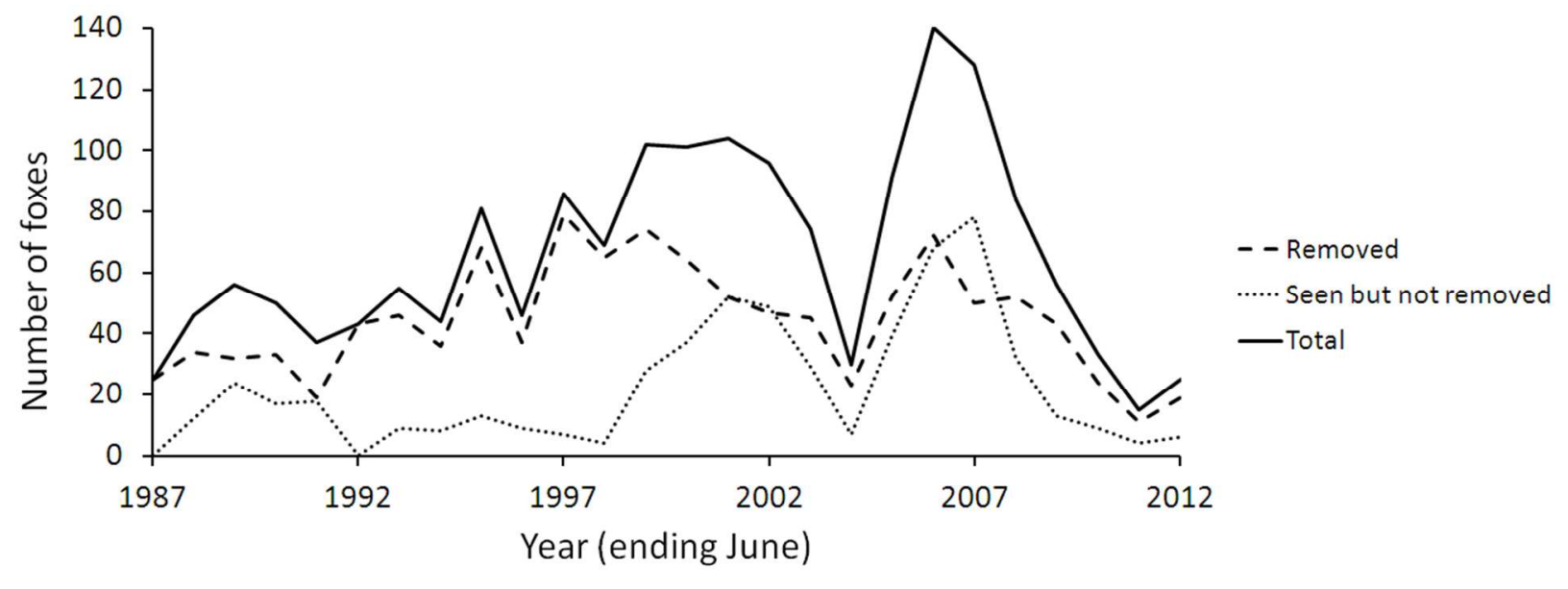

6 Figure 1: The history of the fox eradication campaign on Phillip Island, in terms of a) person-

7 hours of effort invested in each management action, and b) number of foxes detected

8 (removed and sighted). All methods were used to remove foxes, and some foxes were seen but

9 not removed when spotlighting and hunting. 


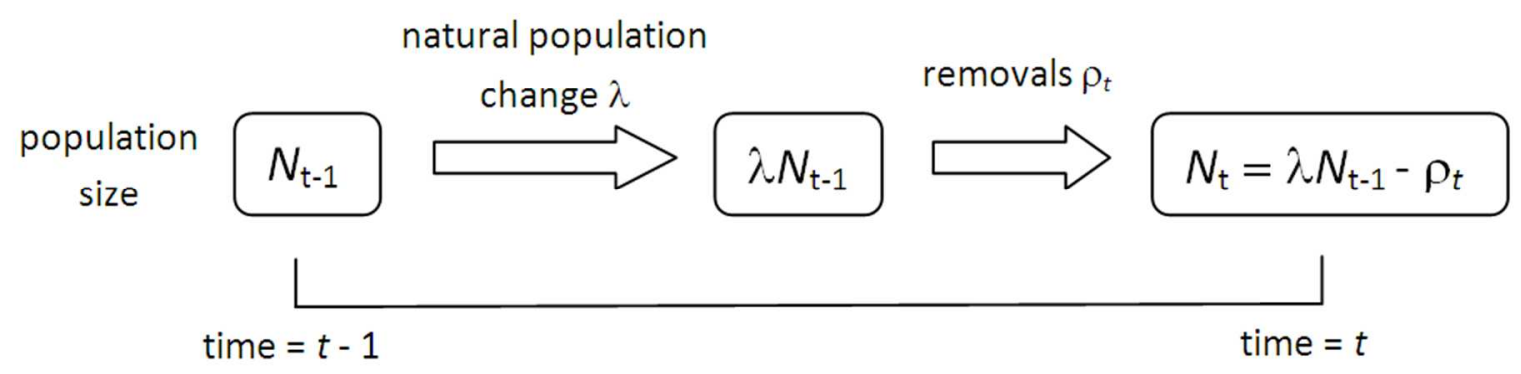

1

2 Figure 2: Order of events in each year within our Bayesian catch-effort model

3

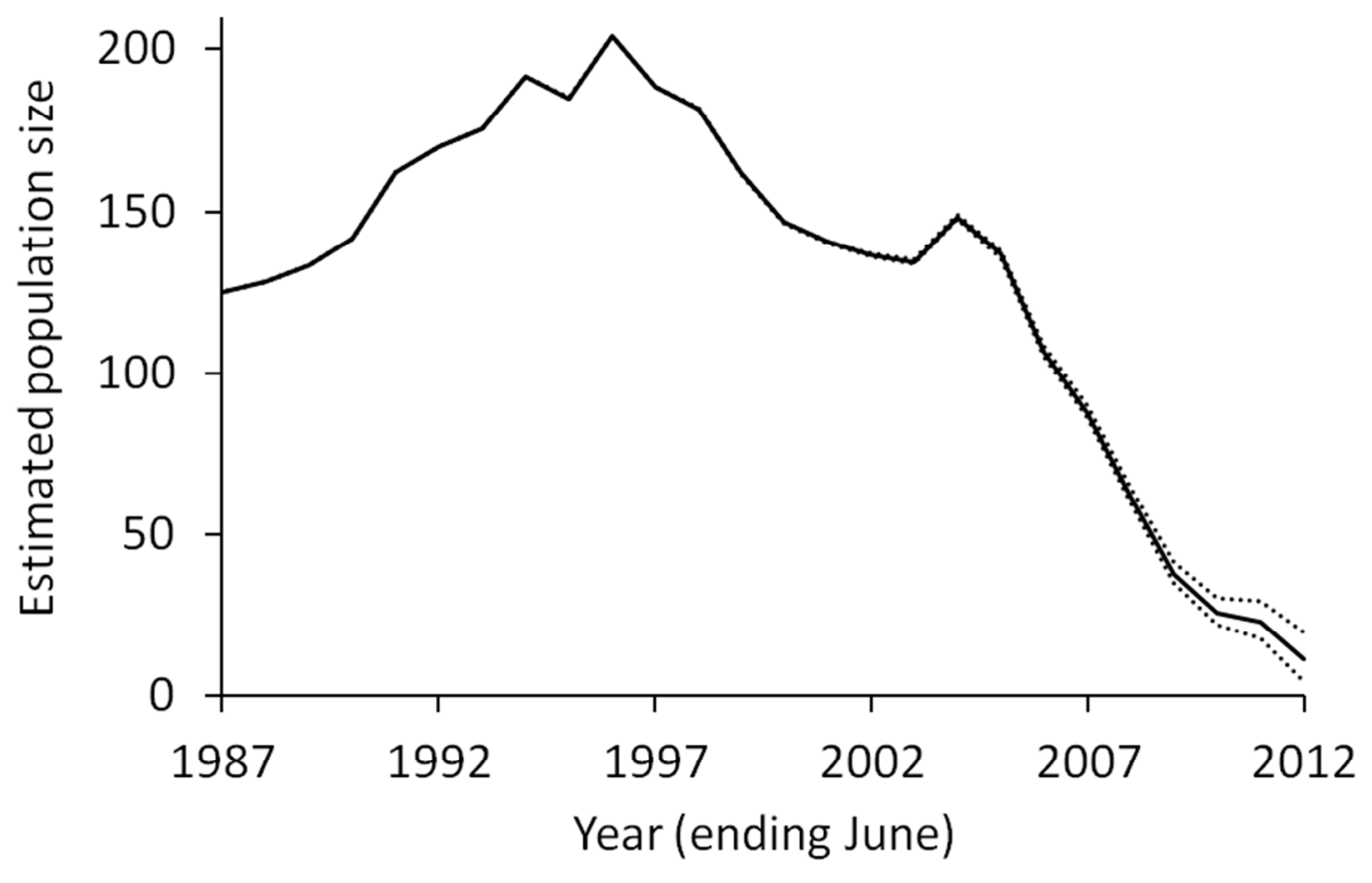

4

$5 \quad$ Figure 3: Estimated population size $N_{t}$ of foxes on Phillip Island through time. Showing mean

6 estimate (solid line) and 95\% credible interval (dotted lines). 
1

2

3

4

5

6

7

8

9

10

11

12

13

14

15

16

17

18

19

20

21

22

23

24

25

26

27

28

29

30

31

32

33

34

35

36

37

38

39

40

41

42

43

44

45

46

47

48

49

50

51

52

53

54

55

56

57

58

59

60

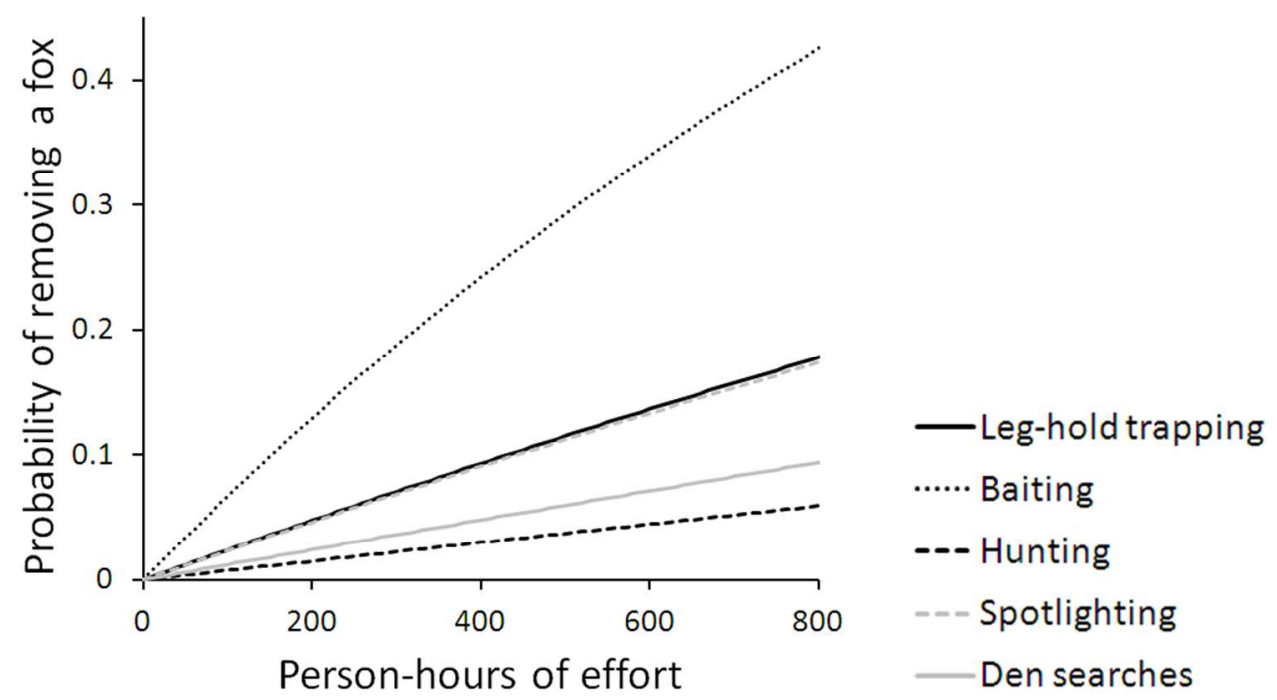

1 a)

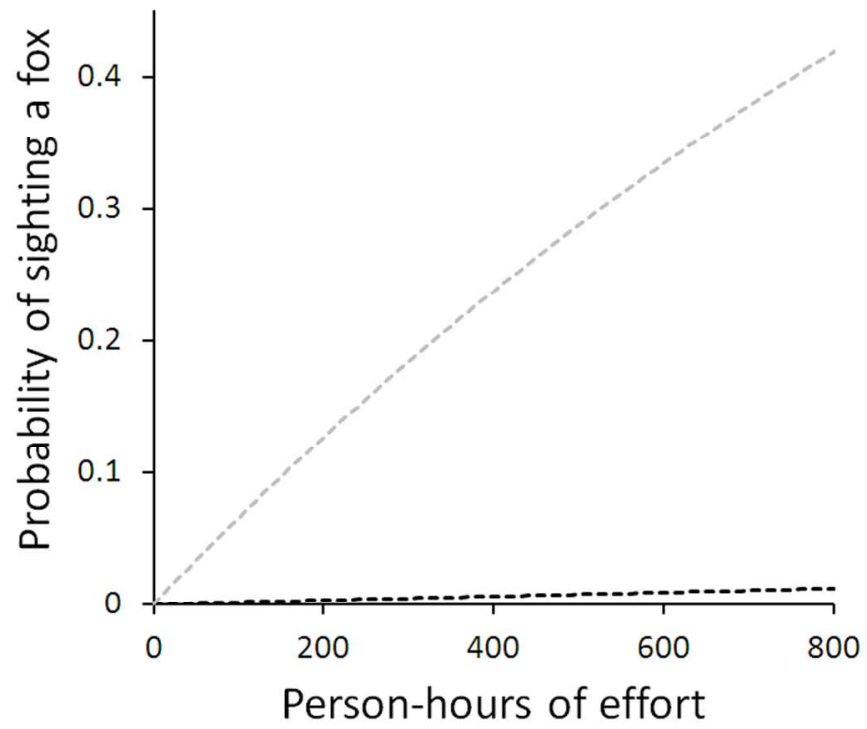

b)

$3 \quad$ Figure 4: Detection curves for the different management methods, showing a) The probability

4 of removing an individual fox, calculated with the mean estimates of $a_{j}$, and b) The probability

5 of sighting an individual fox, calculated with the mean estimates of $b_{j}$. 


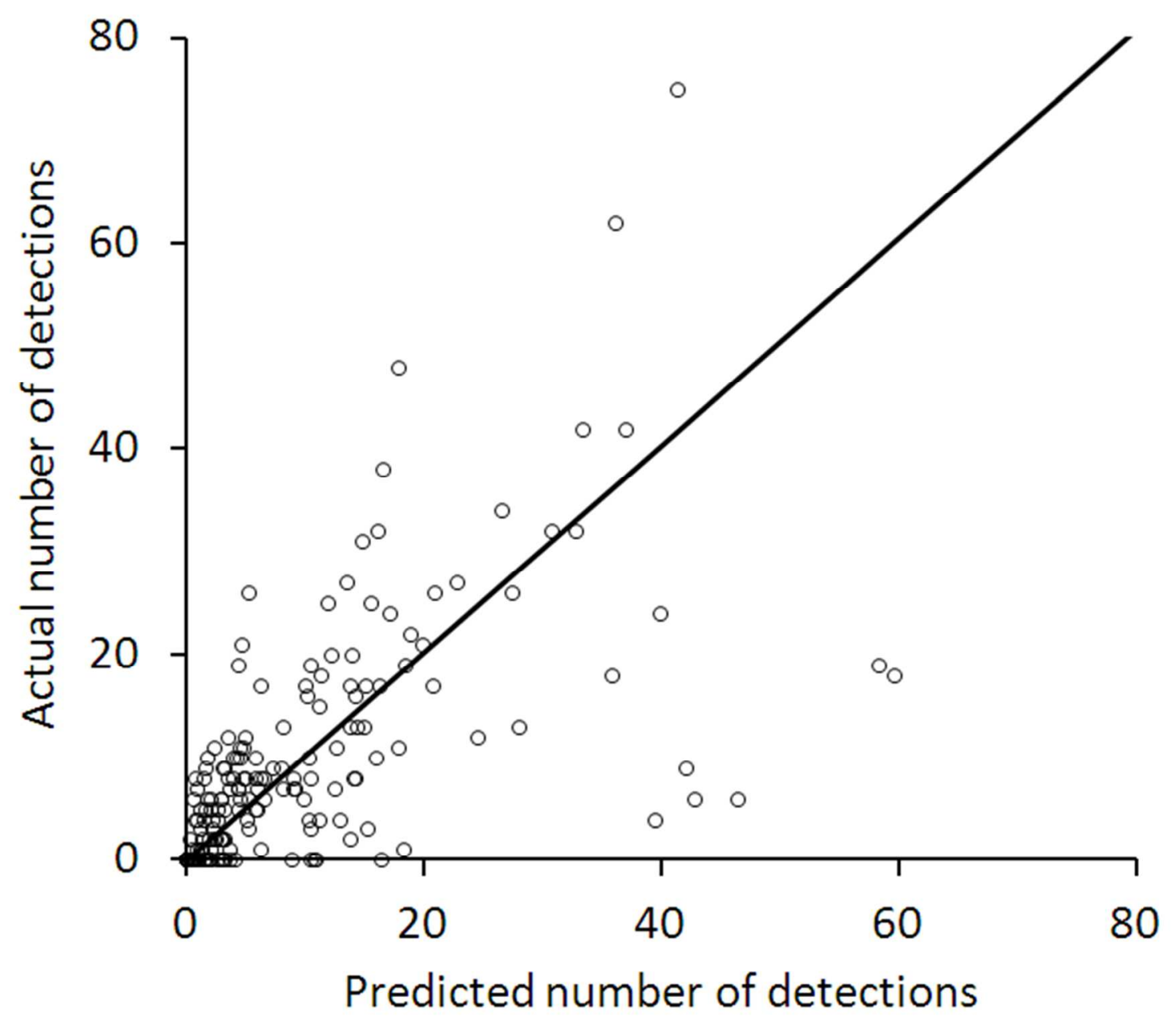

1

2 Figure 5: The model-predicted versus actual number of fox detections (both removals and

3 sightings). Each point represents a single method in a single year. The solid line was fitted using

4 a standardised major axis regression (see text). 


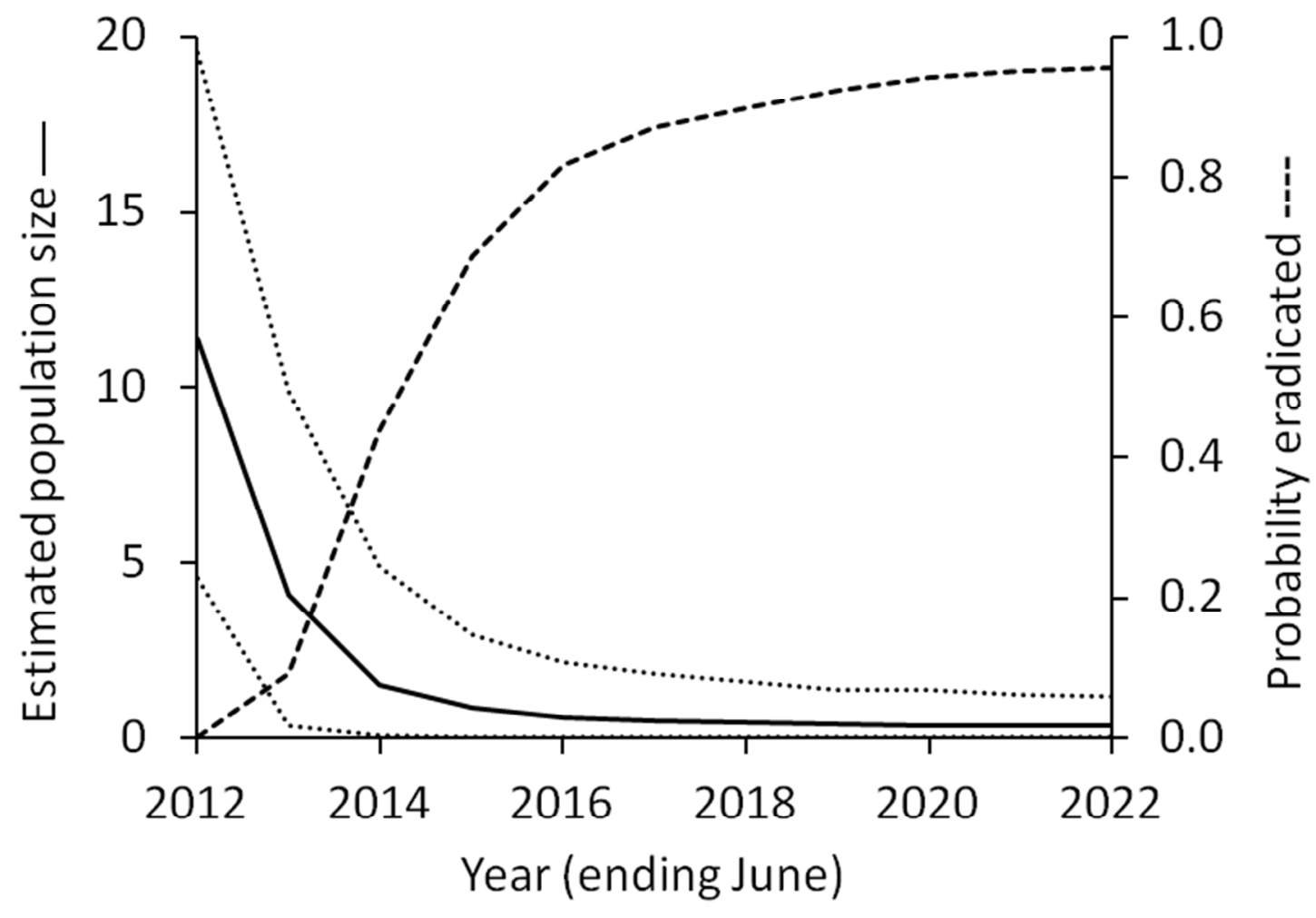

1

2 Figure 6: The estimated fox population size (left axis, solid line $=$ mean, dotted lines $=95 \%$

3 credible interval) and the probability foxes have been successfully eradicated (right axis, dashed

4 line) under a future scenario where management continues but no further foxes are detected.

5 The annual number of person-hours spent on each management method is described in the 6 text. 


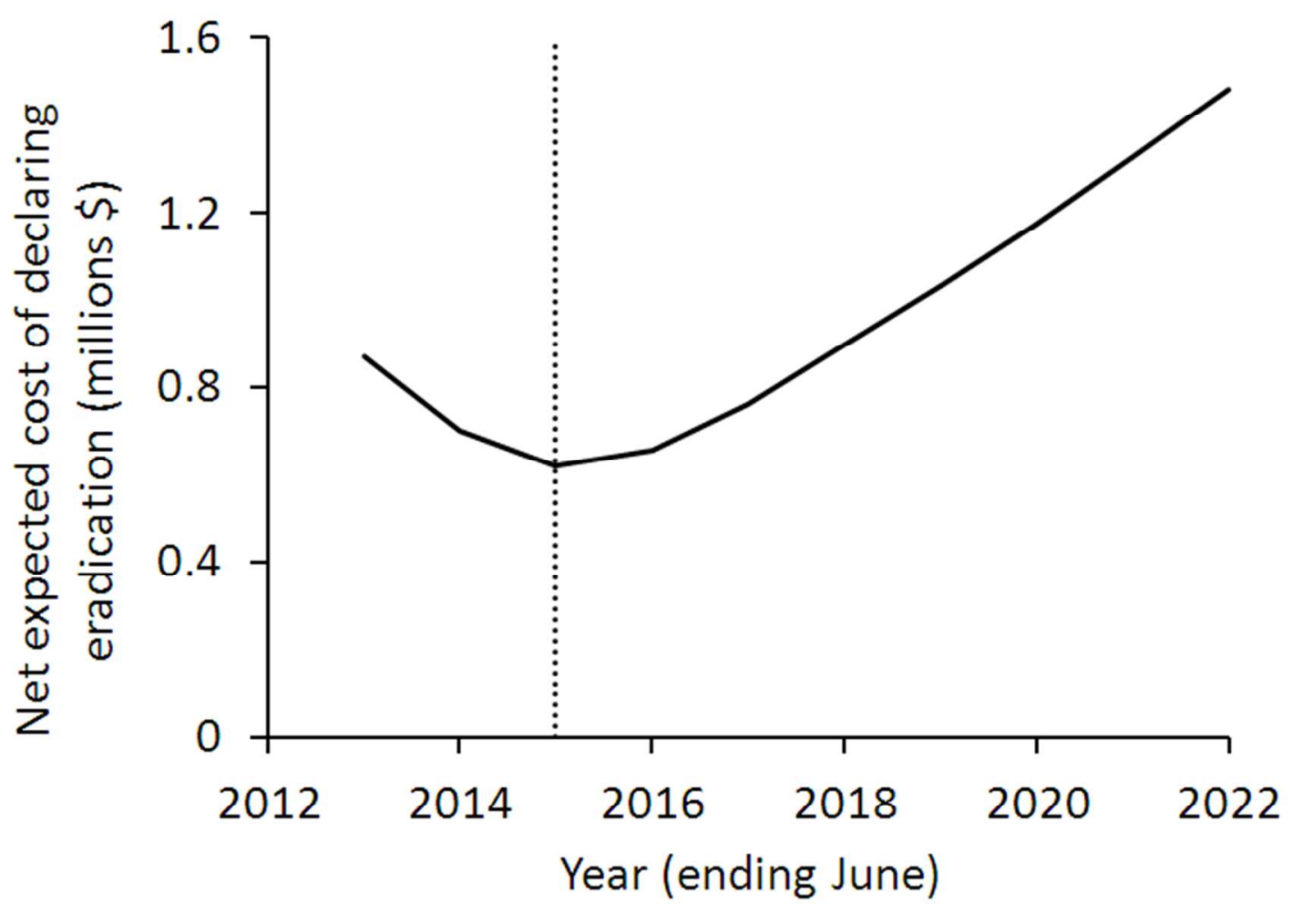

1

$2 \quad$ Figure 7: The net expected cost of declaring eradication in different years (solid line), if

3 management continues as it has been but no further foxes are detected. The current yearly cost

4 of management is $A \cup \$ 160,000$, and we assume the cost of declaring eradication prematurely is

5 six times the current yearly management cost. The optimal year to declare foxes successfully

6 eradicated (dotted line) is 2015, after three years without detection. 


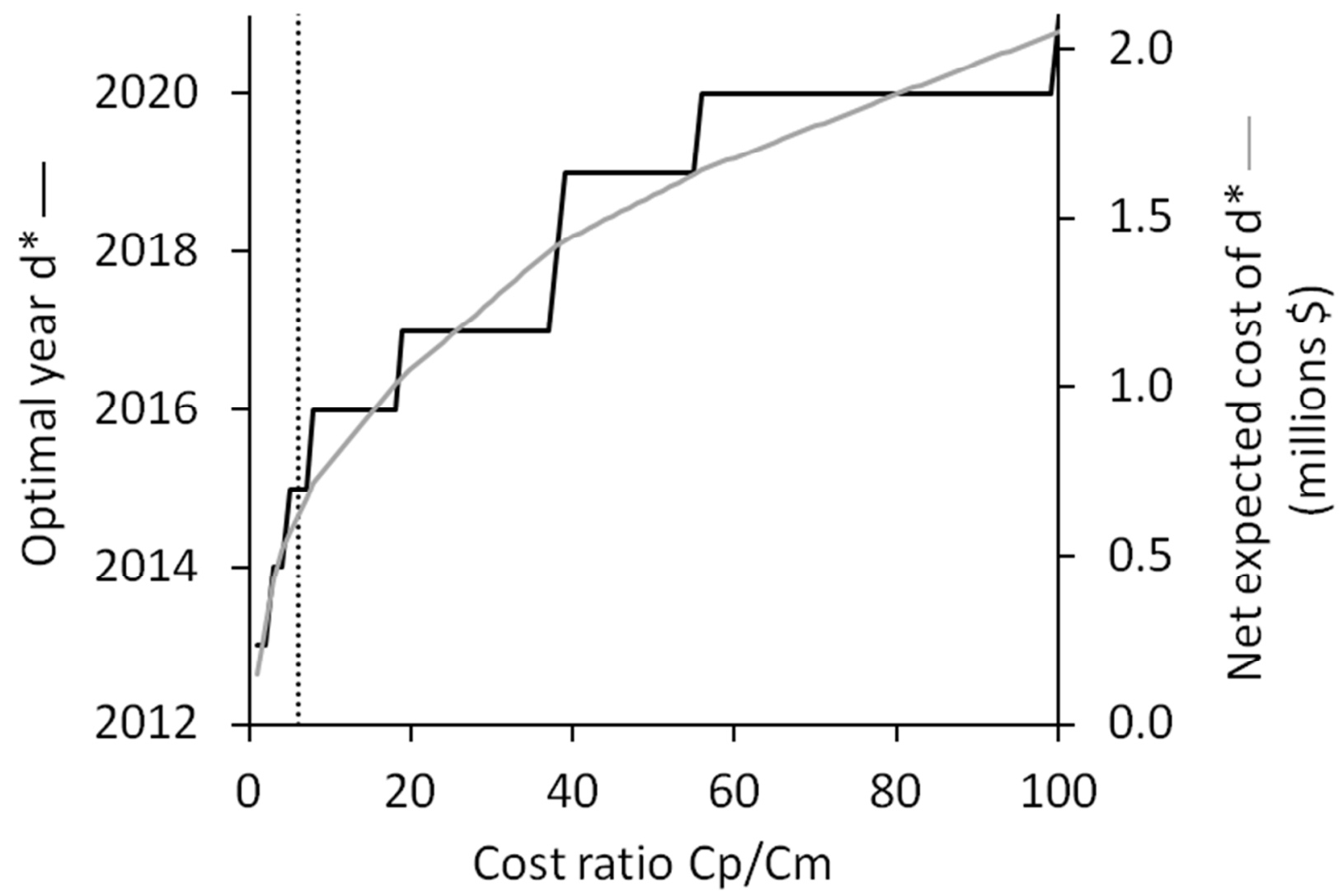

1

2 Figure 8: The optimal year (left axis, solid black line) and the net expected cost of declaring

3 eradication in the optimal year (right axis, solid grey line) for a range of different cost ratios.

4 Our baseline assumption for foxes on Phillip Island (a cost ratio of 6 ) is marked with the black

5 dotted line.

6 


\section{Supplementary Material: OpenBUGs code for Bayesian catch-effort model}

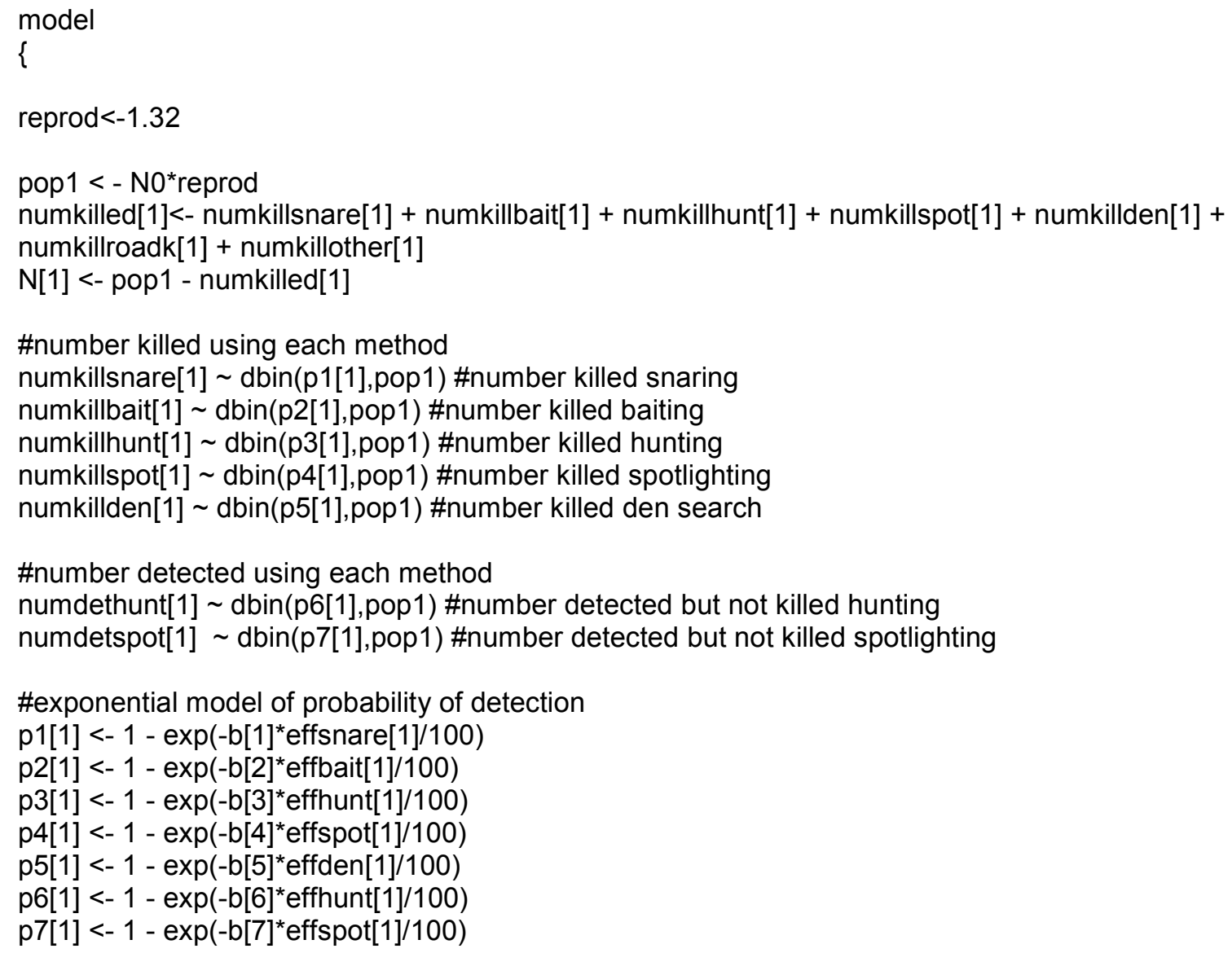


\#number detected using each method

numdethunt[i] dbin(p6[i],pop[i]) \#number detected but not killed hunting

numdetspot[i] dbin(p7[i],pop[i]) \#number detected but not killed spotlighting

\#exponential model of probability of detection

$\mathrm{p} 1[\mathrm{i}]<-1-\exp \left(-\mathrm{b}[1]^{*}\right.$ effsnare[i]/100)

$\mathrm{p} 2[\mathrm{i}]<-1-\exp \left(-\mathrm{b}[2]^{*}\right.$ effbait[i]/100)

p3[i] <- $1-\exp \left(-b[3]^{*}\right.$ effhunt $\left.[i] / 100\right)$

$\mathrm{p} 4[i]<-1-\exp \left(-\mathrm{b}[4]^{*}\right.$ effspot[i]/100)

p5[i] <- $1-\exp \left(-b[5]^{*}\right.$ effden[i]/100)

p6[i] <- $1-\exp \left(-b[6]^{*}\right.$ effhunt $\left.[i] / 100\right)$

p7[i] <- $1-\exp \left(-b[7]^{*}\right.$ effspot[i]/100)

\#

fitkillsnare[i] <- p1[i]* pop1

fitkillbait[i] <- p2[i] ${ }^{*}$ pop1

fitkillhunt[i] <- p3[i] ${ }^{*}$ pop1

fitkillspot[i] <- p4[i]*pop1

fitkillden[i] <- p5[i] ${ }^{*}$ pop1

fitdethunt[i] <- p6[i] ${ }^{*}$ pop1

fitdetspot[i] <- p7[i] ${ }^{*}$ pop1

\}

\#probability of extinction

extinct <- $\operatorname{step}(1-\mathrm{N}[\mathrm{K}])$

\# prior on initial population size NO

N0 dunif $(1,1600)$

\# priors for effectiveness of methods bj

for (i in 1:7) \{

$\mathrm{b}[\mathrm{i}] \sim \operatorname{dunif}(0,10)$

\} 


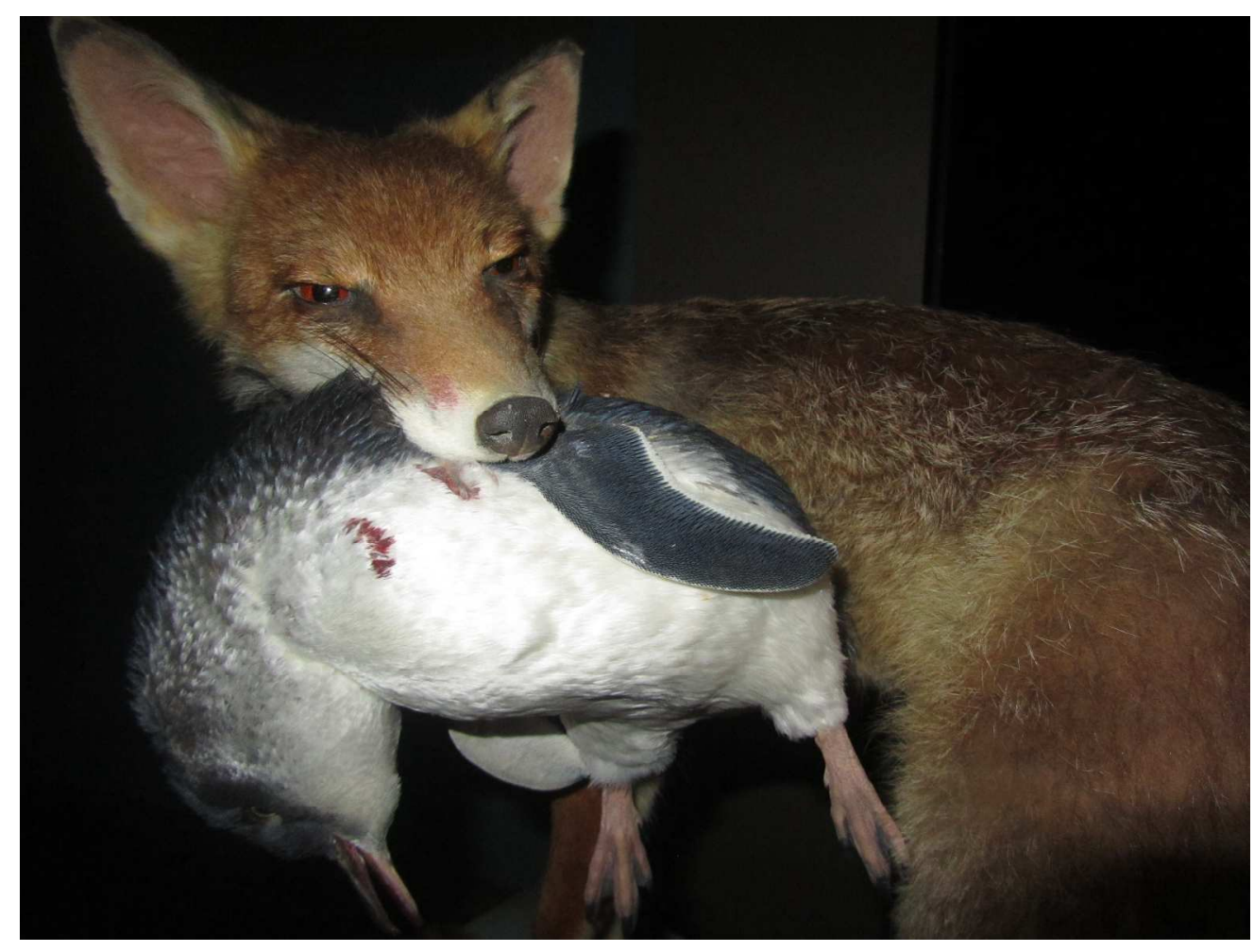

Photo credit: Danene Jones, Phillip Island Nature Parks. $609 \times 457 \mathrm{~mm}(180 \times 180 \mathrm{DPI})$ 